\title{
Dynamic Localization of Vapor Fraction in Turbo Pump Inducers by X-Ray Tomography
}

\author{
Walid Hassan, Samuel Legoupil, Dominique Chambellan, and Stéphane Barre
}

\begin{abstract}
An experimental study of the instantaneous local behavior of cavitation in turbo-pump inducers is presented in this article. Experiments held on a hydrodynamic facility equipped with an Ariane 5 inducer permitted achieving the aim. Cavitation is attained by reducing the pressure in a turbo machine having an inducer rotating at $4000 \mathrm{rpm}$. An x-ray tomography system developed specially for this aim, was used to measure the cavitation. The system employed an industrial $x$-ray generator and scintillation detectors. The generator/detectors system was fixed while the inducer was rotating. Vapor fraction was determined instantaneously, which confirms the applicability and the precision of the method in such type of measurements despite the constraints imposed by the geometry and the rotation speed. The dense composition of the system components introduced difficulties in the measurement especially at the inducer axis. However, prior information concerning the vapor regime as well as its stationary behavior in certain time intervals helped overcome this problem. Consequently a quantitative and qualitative evaluation of the vapor fraction is obtained. Results show a cavitation regime mostly synchronous with the rotation of the inducer thus permitting the use of tomographic reconstruction for the localization of vapor in the machine. An algebraic reconstruction algorithm (ML-EM) was used to achieve image reconstruction.
\end{abstract}

Index Terms-Cavitation, industrial X-ray generators, tomography, turbo pumps.

\section{INTRODUCTION}

$\mathbf{S}^{\mathrm{p}}$ PACE exploration motivates the space industry to develop launchers with more and more efficiency so that they can transmit loads to space. In order to deliver combustibles to the combustion chamber during flight, launchers engage high flow turbo pumps (e.g: $0.5 \mathrm{~m} 3 / \mathrm{s}$ of liquid Hydrogen). Being constrained by size, the inducer-located in the upper stage of the launcher-should rotate at high speeds to maintain a constant supply of fuel (Oxygen and Hydrogen). The high rotational velocity, coupled with the inherently low pressure in the propellant tanks cause cavitation. Many research groups have attempted to illustrate the behavior of air bubbles and cavitation pockets in turbo pumps. [4]-[7]. These methods used gamma-rays from radioactive sources to perform tomographic measurements of the pumps. However, the radiation flux possible from any reasonable source is too low to provide the instantaneous evaluations desired in this application.

W. Hassan, S. Legoupil, and D. Chambellan are with the Commissariat a lénergie atomique, 91191 Gif sur Yvette, France.

S. Barre is with the LEGI-CNRS-INPG, 38041 Grenoble, France.
Recently Stutz et al. [8] adapted an ionizing radiation technique to describe and measure the vapor fraction in a pipe with two-phase flow. An x-ray generator and a set of detectors were employed to achieve the measurement. The efficiency and robustness of the method support its application to explore more complex systems as well as turbo pump inducers. However the use of $\mathrm{x}$-ray generators-especially the industrial types-is still not well reputed in this domain. An important reason for such a bad reputation is that these generators show sharp instabilities in the energy and the number of x-ray photons of the emitted beam [9].

A similar work was carried out by Coutier-Delgosha et al. in 2003 [10]. The aim of their work was to measure the vapor fraction in cavitation pockets produced by water flow on a hydrofoil. Their measurements were verified via numeric calculations obtained by coupling Navier-Stoke equations to a simplified cavitation model.

A research work was carried out at CEA- FRANCE in 2000, where a gamma tomograph was developed to measure the void fraction in a fixed bed petrochemical reactor. The system contained a $300 \mathrm{mCi}$ Cesium source and a group of BGO scintillation detectors. The filtered back-projection method was used to reconstruct images of the distribution of vapor in the reactor [11].

A tomographic system, including both a gamma tomograph and a capacitive tomograph, was developed by Johansen $e t$ al. in 1996 [12]-[14]. This system was used to visualise a three-phase flow containing oil, water and gas. The capacitive tomograph was used to distinguish between the oil and the water, which could not be done with the gamma tomograph due to the small difference between their densities.

Prasser et al. [15] in 2004 worked on a gamma ray tomograph with a source of $5 \mathrm{Ci}$ of Cesium for the visualisation of two-phase flow (liquid and air) in the inducer of an axial pump. Images were reconstructed with the filtered back-projection algorithm, from measurements averaged over 7 minutes.

Methods using gamma rays showed good results in measuring and visualizing multi-phase flows. However, due to the fact that these methods can't perform high speed measurements, we report in this work the use of an x-ray generator to perform tomographic measurements on a turbo pump. Indeed the system takes into account the instabilities in the $\mathrm{x}$-ray beam, the $3 \mathrm{D}$ geometry of the inducer, the high rotation speed, and the dynamic nature of the vapor structures.

\section{NOMENCLATURE}

- $\beta$ : Vapor fraction.

- $\sigma$ : The cavitation parameter or the similarity parameter for cavitation [1]. 


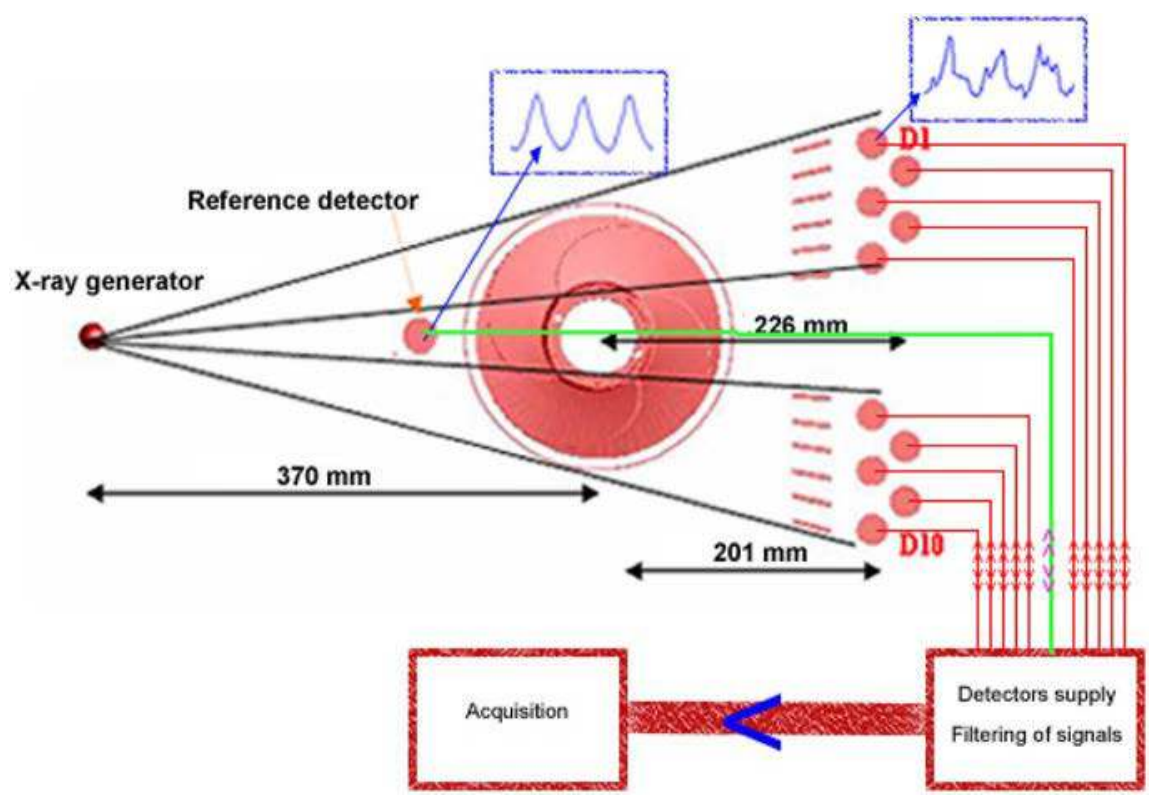

Fig. 1. The measurement system.

- $I_{0}$ : Calibration measurement when no liquid is in the machine $(\beta=1)$.

- $I_{1}$ : Calibration measurement when the machine is full of water $(\beta=0)$.

- $I$ : Measurement with a vapor faction $\beta$ to be estimated.

- $I_{\text {no }}$ : X-rays intensity received by the detector when no object is between the source and the detector.

- $I_{\text {ref }}$ : A reference factor that indicates the level of the flux [Energy, Number of photons] of the X-ray beam.

- $\mu$ : Signifies the attenuation coefficient $\mathrm{cm}^{-1}$.

- $\rho$ : The density in $\mathrm{g} . \mathrm{cm}^{-3}$.

- $x$ : Thickness in $\mathrm{cm}$.

- Indices:

— pw: Housing walls.

- mi: Inducer metal.

- W: Water.

$-\mathrm{v}$ : Vapor.

- d: Detector number

$-\theta$ : Angular position of the inducer.

\section{METHOD}

Tomography was achieved by immobilizing the source-detector measurement system while rotating the inducer. Projections were acquired by 10 detectors for a total of 75 angles of incidence (Fig. 1). Measurements could not be acquired in the center of the machine due to the presence of the mounting axis holding the inducer, which constitutes $35 \mathrm{~mm}$ of iron. This quantity of iron is capable of absorbing all incident X-ray photons in the energy range in which we are working.

The x-ray absorption technique is used to perform integral measurements of the vapor volume in the inducer. We define the vapor fraction, $\beta$, as the ratio of the volume of vapor to the total volume of measurement :

$$
\beta(t)=\frac{\text { Volume of vapor }(t)}{\text { Total measurement volume }} .
$$

The vapor fraction could be estimated from the three different measurements $I_{0}($ bet $a=1), I_{1}($ bet $a=0)$ and $I($ bet $a)$ as listed below :

$$
I_{0}=I_{\mathrm{no}} \cdot \exp \left(-\mu_{m h} \cdot \rho_{m h} \cdot x_{m h}\right)
$$

where $\mu_{m h} \cdot \rho_{m h} \cdot x_{m h}=\mu_{p w} \cdot \rho_{p w} \cdot x_{p w}+\mu_{m i} \cdot \rho_{m i} \cdot x_{m i}$.

$$
\begin{gathered}
I_{1}=I_{\mathrm{no}} \cdot \exp \left(-\mu_{W} \cdot \rho_{W} \cdot x_{W}-\mu_{m h} \cdot \rho_{m h} \cdot x_{m h}\right) \\
\text { and : } \\
I=I_{\mathrm{no}} \cdot \exp \left(-\mu_{M} \cdot \rho_{M} \cdot x_{M}-\mu_{m h} \cdot \rho_{m h} \cdot x_{m h}\right)
\end{gathered}
$$

where $\mu_{M} \cdot \rho_{M} \cdot x_{M}=\mu_{W} \cdot \rho_{W} \cdot x_{W} \cdot(1-\beta)+\mu_{v} \cdot \rho_{v} \cdot x_{v} \cdot(\beta)$. Because $\rho_{v}$ is negligible compared to $\rho_{W}$ we can write :

$$
\mu_{M} \cdot \rho_{M} \cdot x_{M}=\mu_{W} \cdot \rho_{W} \cdot x_{W} \cdot(1-\beta) .
$$

Solving the equations for beta we get:

$$
\beta=1-\frac{\ln \left(\frac{I_{0}}{I}\right)}{\ln \left(\frac{I_{0}}{I_{1}}\right)} .
$$

In fact, the thickness of metal varies with the rotation of the inducer. Thus the calibration measurements should be performed for all angular incidences of the inducer. Hence $\beta$ is also a function of the angle and is given by:

$$
\beta(\theta)=1-\frac{\ln \left(\frac{I_{0}(\theta)}{I(\theta)}\right)}{\ln \left(\frac{I_{0}(\theta)}{I_{1}(\theta)}\right)}
$$

where $\theta$ indicates the angular position of the inducer. 


\section{A. Test Facility and Measurement System}

Experiments were performed on the water test bench of the Centre de Recherche et d'Essais de Machines Hydrauliques de Grenoble [CREMHyG]. The bench is equipped with a special Aluminum inducer designed by Snecma, the company in charge of the Ariane space manufacturing.

Cavitation is produced by reducing the inlet pressure of the pump, while maintaining the inducer rotation speed at 4000 rpm. The cyclo-stability of cavitation structures is required in order to perform image reconstruction later. The angular position of the inducer is provided by a position sensor situated at the housing of the machine. This sensor provides a signal between 0 and 10 volts corresponding to angular positions between 0 and 360 degrees. Cavitation measurements were performed in three axial positions at the machine, with a section thickness of 20 $\mathrm{mm}$ for each axial position.

A Philips M160 X-ray generator and 11 NaI scintillation detectors functioning in current mode were employed to perform integral measurements. The X-ray tube high voltage supply was fixed at $80 \mathrm{kV}, 30 \mathrm{~mA}$ for $I$ and $I_{1}$ measurements while $I_{0}$ is performed at $80 \mathrm{kV}, 5 \mathrm{~mA}$ to avoid saturating the detectors. Detector positions were selected based on the opening angle of the beam from the x-ray tube. Unfortunately, due to the presence of support equipment for the pump, the same detector source spacing could not be used for all of the detectors.

Technical parameters of the system (high voltage, current, detection area, etc.) were optimized to ensure a good signalnoise ratio and thus to reduce the measurement error. Simulation codes like VXI [17] and Macalu 7 [18] were used for this purpose. Practically the measurement error is inversely proportional to x-ray intensity (defined by the tube current) and the measurement time. Because the high rotational speeds limit the measurement time, we used the maximum tube current at the lowest acceptable tube high voltage to maximize the contrast.

Signals issuing from $\mathrm{X}$-ray detectors as well as signals from the position sensor, and pressure sensors situated at the housing of the machine were acquired simultaneously. The acquisition was performed with a National Instruments [NI] acquisition card (PCI 6033) using Labview. The acquisition frequency is $5000 \mathrm{~Hz}$ at each detector, thus permitting to acquire 75 projections during one complete rotation of the inducer. This reflects a measurement time in the order of $200 \mu \mathrm{sec}$ per projection.

Fig. 1 shows the presence of a reference detector situated between the X-ray tube and the machine. The purpose of this detector is to monitor the X-ray beam, and the acquired signal is used for treating instabilities in the $\mathrm{X}$-ray beam. These fluctuations are related to the high voltage generator of the $\mathrm{x}$-ray tube.

The procedure to eliminate the fluctuations has been previously described [7]. The main idea is to perform a double calibration in order to determine the vapor fraction $(\beta)$ : one for the angular position $(\theta)$, and another for the variations of energy and flux $\left(I_{\text {ref }}\right)$ provided by the reference detector. Thus $\beta$ can be rewritten as follows:

$$
\beta(\theta)=1-\frac{\ln \left(\frac{I_{0}\left(\theta, I_{\mathrm{reI} I}\right)}{I\left(\theta, I_{\mathrm{reI} I}\right)}\right)}{\ln \left(\frac{I_{0}\left(\theta, I_{\mathrm{reI} \Gamma}\right)}{I_{1}\left(\theta, I_{\mathrm{reI}}\right)}\right)} .
$$

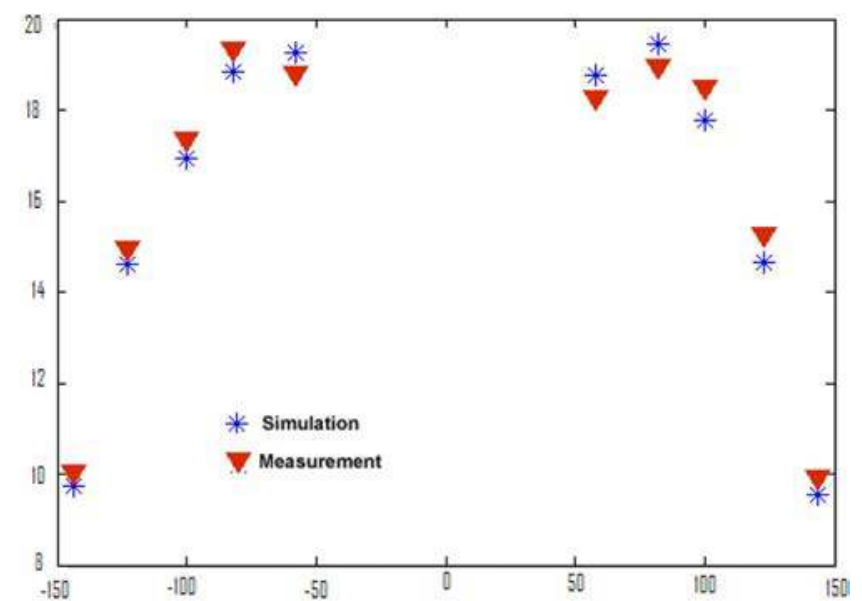

Fig. 2. The water thickness obtained. The blue markers are the values determined by simulation and the red ones are those measured.

Therefore, the void fraction measured at a time $t$, in an angular position $\theta$, by a detector $d$ is given by:

$$
\beta^{d}(\theta, t)=1-\frac{\ln \left(\frac{I_{0}^{d}\left(\theta, I_{\mathrm{re},}, t\right)}{I^{d}\left(\theta, I_{\mathrm{reI},}, t\right)}\right)}{\ln \left(\frac{I_{n}^{d}\left(\theta, I_{\mathrm{re} \Gamma}, t\right)}{I_{1}^{d}\left(\theta, I_{\mathrm{rel}}, t\right)}\right)}
$$

\section{B. Experimental Validation}

The experimental validation of the measurements was performed by analysing two different parameters. The first is concerned with finding $I_{0}, I_{1}$ and the other is for the determination of the void fraction. The thickness of the measurement volume seen by each detector can be determined via the calibration measurements $I_{0}$ and $I_{1}$ as follows:

The measurement $I_{0}$ can be written as:

$$
I_{0}^{d}(\theta)=I_{\mathrm{no}}^{d} \exp \left(-\mu_{m h} \cdot \rho_{m h} \cdot x_{m h}^{d}(\theta)\right)
$$

while $\mu_{m h} \cdot \rho_{m h} \cdot x_{m h}^{d}(\theta)=\mu_{p w} \cdot \rho_{p w} \cdot x_{p w}^{d}(\theta)+\mu_{m i} \cdot \rho_{m i} \cdot x_{m i}^{d}(\theta)$ The measurement $I_{1}$ is expressed as follows:

$$
\begin{aligned}
I_{1}^{d}(\theta)=I_{\text {no }}^{d} \exp ( & -\mu_{W} \cdot \rho_{W} \cdot x_{W}^{d}(\theta) \\
& \left.-\mu_{m h} \cdot \rho_{m h} \cdot x_{m h}^{d}(\theta)\right) .
\end{aligned}
$$

Solving for the water thickness from the two equations we get:

$$
x_{W}^{d}(\theta)=\left(\frac{1}{\mu_{W} \cdot \rho_{W}}\right) \ln \left(\frac{I_{0}^{d}(\theta)}{I_{1}^{d}(\theta)}\right) .
$$

Note that in this equation we did not consider $I_{\text {ref }}$ due to the fact that $I_{0}$ and $I_{1}$ could be averaged with respect to the angular position for a long time and thus we eliminated the fluctuation effect.

However $\mu_{W} \cdot \rho_{W}$ in the equation were measured experimentally by determining the attenuation of a $10 \mathrm{~mm}$ sheet of Plexiglas, knowing that this is equivalent to $12 \mathrm{~mm}$ of water in terms of attenuation. Fig. 2 shows the measurement volume thickness 


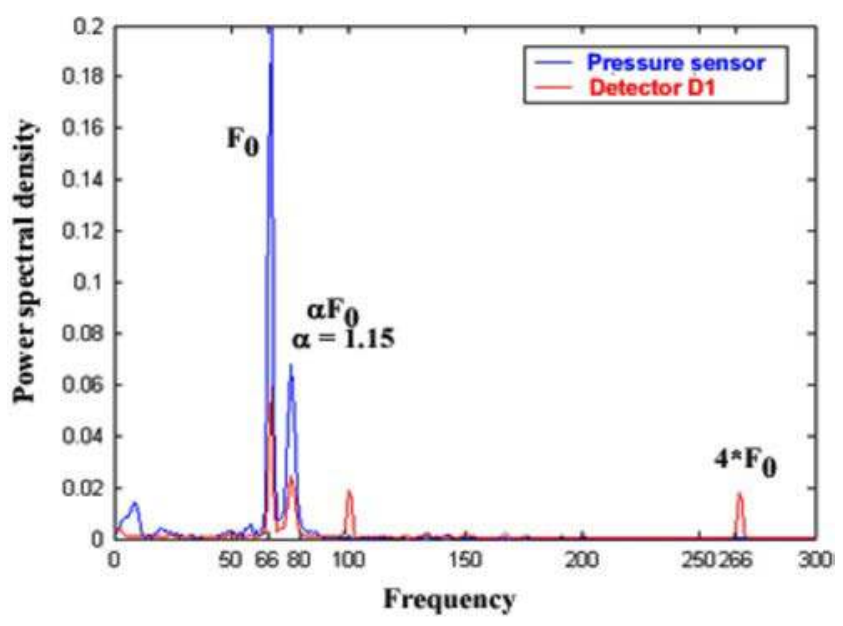

Fig. 3. The spectral analysis for measured vapor fraction and the pressure sensor.

obtained experimentally compared to that obtained by simulations. Values seen by the 10 detectors at a fixed angular position are also shown.

The second validation procedure is to compare frequency analysis (spectrum) of the measured vapor fraction to the spectrum of the pressure sensor situated at the housing of the machine, provided that the pressure sensor is sensible to vapor structures produced in the machine.

Fig. 3 shows an example of spectra obtained from both the pressure sensor and the vapor fraction measured by an X-ray detector. The spectra in the figure correspond to a super synchronous cavitation regime. In this regime usually we find two essential forms of cavitation : pockets rotating at the inducer frequency $F_{0}$ and pockets rotating at a superior frequency $\alpha \times F_{0}$. The two sensors show well the spectral content of this cavitation mode. The super synchronous term $\left(1.15 \times F_{0}\right.$, where $F_{0}=66.7$ $\mathrm{Hz}$ is the inducer rotation frequency) could also be seen by the two sensors. The X-ray sensor is still sensitive to small structures alternating at $4 \times F_{0}$ that could not be seen by the pressure sensor.

The analysis of the X-ray detector spectrum always showed a small factor of $100 \mathrm{~Hz}$ caused by the fluctuations of the X-ray beam. However, this was caused by the measurement $I_{0}$, rather than by a defect in the treatment method. As mentioned above, $I_{0}$ is performed at $5 \mathrm{~mA}$ while $I_{1}$ and $I$ were performed at 30 $\mathrm{mA}$ in order to avoid saturation of the detectors. The compensation is done by multiplying $I_{0}$ by a factor (6) assuming linearity in the number of emitted photons as a function of the tube current. This hypothesis is true in case we don't have instabilities in the X-ray beam, especially it has been shown [7] that fluctuations are more intense at $30 \mathrm{~mA}$ than at $5 \mathrm{~mA}$. A thorough analysis of this problem shows that the fluctuations are more related to the energy rather than the number of photons, which permits such a compensation to take place.

In the mean time the $100 \mathrm{~Hz}$ factor is accepted since the amplitude of these fluctuations is too small, to affect the measurements. Furthermore the image reconstruction method is still robust while the noise level (beam fluctuation) is lower than $15 \%$
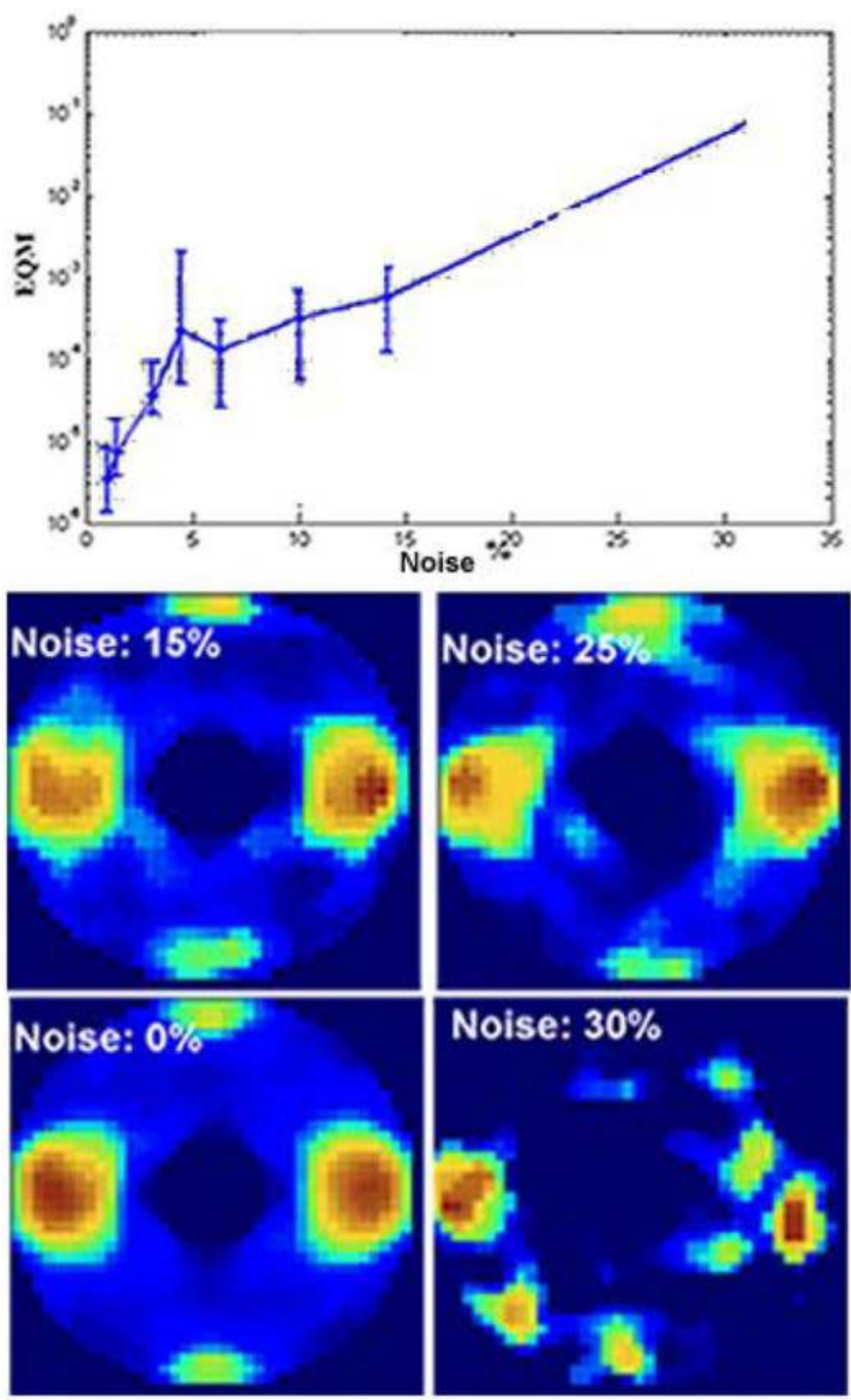

Fig. 4. The mean square deviation as a function of the noise level.

in the measurements. This was proved by performing reconstruction on simulated data and by adding random noise to this data. Fig. 4 shows the mean square deviation of the recontructed image as a function of the noise level in the simulated data. Here a simulated data representing an image with 4 vapor pockets is reconstructed while adding different levels of random noise to these data and then calculating the mean square deviation between the reconstructed image and the real one.

As can be seen in the figure, the reconstruction method is robust upto a noise level of $15 \%$ in the data to be reconstructed.

\section{Void Fraction}

The void fraction is measured at three axial positions on the inducer. At each position, three cavitation cycles corresponding to three different flow rates $(\mathrm{Q} / \mathrm{Qn}=0.9,1.0,1.1)$ were performed. During each cycle, 10 to 15 pressure points were tested. At each pressure point, signals are acquired during $10 \mathrm{sec}$.

A statistical study is performed on the measurements to verify the statistical convergence of the void fraction. Fig. 5 shows the mean void fraction obtained from $\mathrm{N}$ samples divided by 


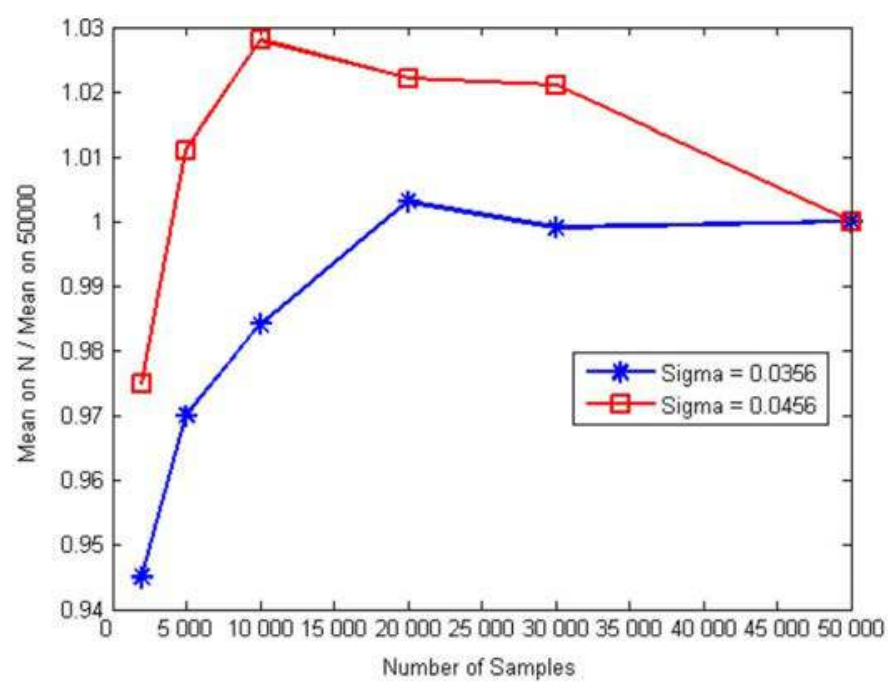

Fig. 5. Statistical convergence of mean void fraction.

the average value obtained from the total number of samples ( 50000 points $=5000 \mathrm{~Hz} * 10 \mathrm{sec})$. The importance of this test is that it defines the threshold of convergence, i.e., the number of samples necessary to ensure the stability of void fraction in vapor structures, noting that stability is necessary to perform the tomographic reconstruction.

Assuming a 3\% uncertainty, we found that the means are convergent after 5000 samples, which corresponds to a $1 \mathrm{sec}$ acquisition time.

\section{TOMOgRaphic MEASUREMENTS}

In order to realize tomographic reconstruction of a section of an object, it is necessary to have projections (or integral measurements) in different angles of incidence. In our case the object to be identified (vapor structures) is dynamic having a behavior that might not be related to that of the inducer. So we might have vapor structures rotating faster (super synchronous) or slower (under synchronous) than the inducer or at the same speed (synchronous). Thus we need to reconstitute the sinograms of measurements.

\section{A. Constitution of Sinograms}

The procedure of the constitution of the sinogram depends on the cavitation mode. So for synchronous modes, there is no specific treatment needed to reconstitute the sinogram. The 75 projections measured by the 10 detectors during a rotation of the inducer are taken completely. While for non synchronous modes two cases could be showed. The first case is that if the periodicity of structures is known from literature, the sinogram could be reconstituted from a certain number of rotations, by taking one projection line per rotation (Fig. 6). The position (delay) of the line to be taken is defined throughout the periodicity.

In the other case, where the periodicity is unknown, we would refer to the spectral analysis of the measured vapor fraction or that of the pressure sensor to determine the periodicity.

\section{B. Reconstruction}

The nature of data contained in the sinograms play an important role in the choice of the reconstruction algorithm. As

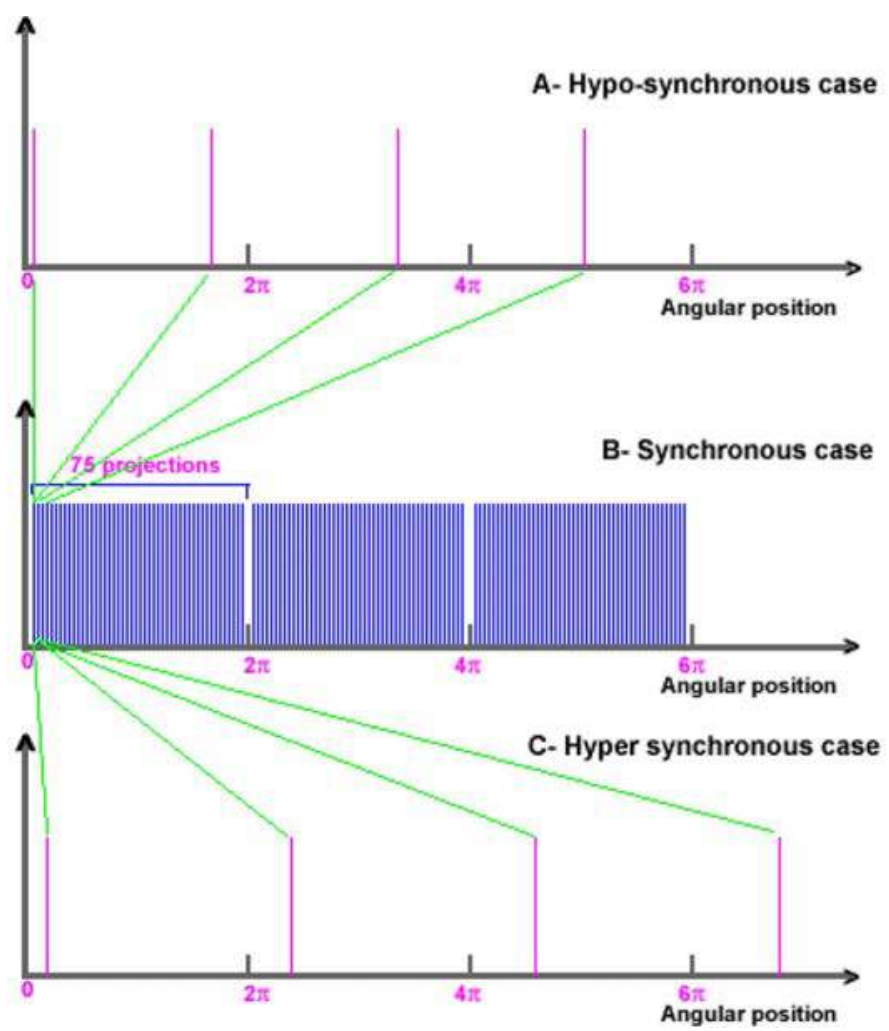

Fig. 6. Constitution of sinograms. For synchronous modes the 75 projections acquired by each detector during on rotation of the inducer are taken into account. For non synchronous modes one projection line per rotation is taken depending of the periodicity of structures.
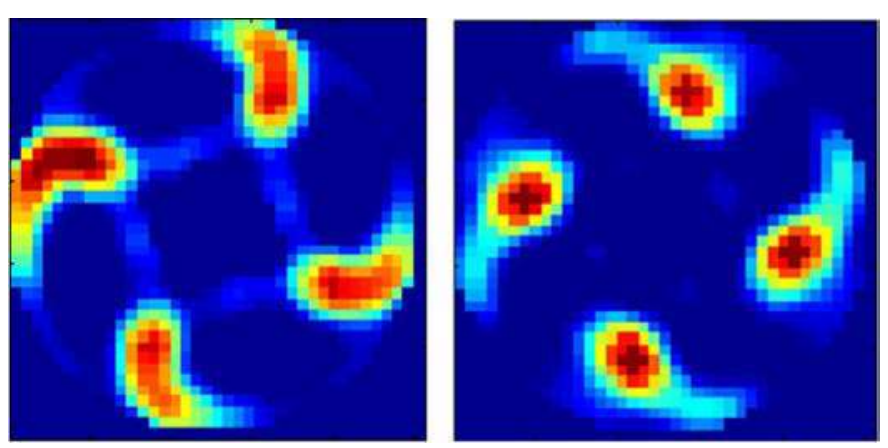

Fig. 7. Reconstructed images corresponding to two different cavitation conditions (Left $\sigma=0.0356$ ) and (Right $\sigma=0.0116$ ).

mentioned above, detectors were placed in a manner to avoid the mounting axis of the machine. Therefore sinograms are truncated at the middle, thus representing an important discontinuity in the projection function. This discontinuity did not permit the use of classical reconstruction methods (analytic ones). For this reason, the well adapted Maximum likelihood expectation maximization (MLEM) statistical method is used [2], [3].

Fig. 7 shows two reconstructed images corresponding to two different sigma values or cavitation conditions $\sigma=0.0356$ and $\sigma=0.0116$ (synchronous regimes) and to a nominal flow $\left(Q / Q_{n}=1\right)$. In both of the reconstructed images we could distinguish the four structures attached to the blades of the inducer. For $\sigma=0.0356$, usually we find cavitation pockets at the boundaries of the blades attached to small marginal structures. 

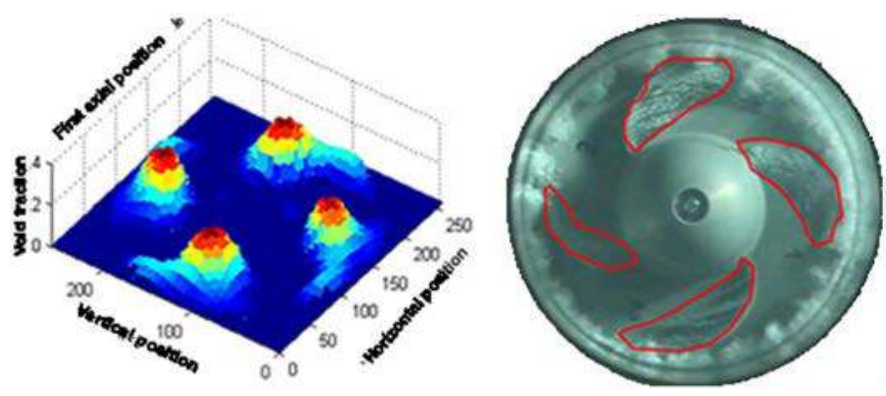

Fig. 8. The reconstructed images for $\sigma=0.0356$ compared to a photographic image taken in the same cavitation conditions.

However this is well reflected by the reconstructed image. In the center of the image we could notice small vapor structures that are attached to the mounting axis of the inducer. In fact we are considering these figures because the reconstructed image refers to data measured in the first axial position or the outermost section of the inducer. In order to verify these results we compare in Fig. 8 a 2D representation of the reconstructed image to a photographic image taken under the same cavitation conditions.

The values of the vapor fraction in the image are re-dimensioned (multiplied by a constant factor). In the photographic image we note the existence of vapor at the walls (marginal cavitation). This is due to the fact that the photos are taken along the axis of rotation, and the vapor that appears in this region of the photo is out of our section of measurement.

\section{CONCLUSION}

The vapor fraction in the machine was measured despite technical difficulties related to the complex geometric design of the inducer and its high rotation speed. Adequate procedures were developed to treat fluctuations in the measurements due to X-ray beam instabilities.

Integral measurements of void fraction achieved at 75 angles of incidence, permitted to determine via tomographic reconstruction the distribution of vapor in the machine. Reconstructed images show good agreement with photographic images taken in identical cavitation conditions.

Improvement of the axial resolution by increasing the number of axial sections to be tested would permit us to reconstruct the vapor fraction distribution in vapor pockets attached to the blades of the inducer.

\section{ACKNOWLEDGMENT}

The authors would like to thank the CNES and SNECMA motors, Rocket Engine Division, the financial supporters of this project, and special thanks to Dr. G. Albano (CNES) and
Dr. P. A. Lambert (SNECMA) for the management and the support of this research.

\section{REFERENCES}

[1] Y. Lecoffre, La Cavitation: Traqueurs de Bulles.. London, U.K.: Hermes Sciences, 1994, ISBN: 286601409X.

[2] L. Shepp and Y. Vardi, "Maximum likelihood reconstruction for emission tomography.," IEEE Trans. Med. Imag., vol. MI-1, no. 2, pp. 113-122, Oct. 1982.

[3] K. Lange and R. Carson, "EM reconstruction algorithms for emission and transmission tomography," J. Comput. Assist. Tomogr., vol. 8, pp. 306-316, 1984

[4] B. Stutz, "Analyse de la structure diphasique et instationnaire de poches de cavitation," Thèse de Doctorat, de L'institut Nationale Polytechnique de Grenoble, Grenoble, France, 1996.

[5] J. De Bernardi, "Aspects expérimentaux et théoriques des instabilités de cavitation dans les yurbopompes des moteurs de fusée.," Thèse de Doctorat, de L'institut Nationale Polytechnique de Grenoble, Grenoble, France, 1996

[6] G. Boitel, Effet Thermodynamique Dans un Inducteur Cavitant Rapport d'avancement de thèse CNRS/CNES/SNECMA, LEGI, Mar. 2005.

[7] W. Hassan, S. Legoupil, S. Barre, and C. Rebattet, "Dynamic vapor fraction measurement in turbopump inducers by X-rays.," in Proc. ASME Fluids Engineering Division Summer Meeting and Exhibition, Houston, TX, Jun. 19-23, 2005

[8] B. Stutz, S. Legoupil, and R. Woo, "Measurements within cloud cavitation by means of x-ray attenuation device," in Proc. ASME Fluids Engineering Division Summer Meeting and Exhibition, Montreal, QC, Canada, Jul. 14-18, 2002.

[9] W. Hassan, S. Legoupil, D. Chambellan, and S. Barre, "Industrial x-ray generators measurements in multi-phase flows," 2005, submitted for publication

[10] O. Coutier-Delgosha, J. F. Devillers, T. Pichon, A. Vabre, R. Woo, and S. Legoupil, "Internal structure and dynamics of sheet cavitation," ENSTA Rep., 2003.

[11] C. Boyer and B. Fanget, "Measurement of liquid flow distribution in trickle bed reactor of large diameter with a new gamma ray tomography system," Chem. Eng. Sci., vol. 57, pp. 1079-1089, 2002.

[12] G. A. Johansen, T. Froystein, B. T. Hjertaker, and O. Olsem, "A dual sensor flow imaging tomographic system," Meas. Sci. Technol., vol. 7, pp. 297-307, 1996.

[13] E. Abro and G. A. Johansen, "Void fraction and flow regime determination by low-energy multi-beam gamma-ray densitometry," in Proc. 1st World Congress Industrial Process Tomography, Buxton, Greater Manchester, U.K., Apr. 14-17, 1999

[14] E. Abro, V. A. Khoryakov, G. A. Johansen, and L. Kocbach, "Determination of void fraction and flow regime using a neural network trained on simulated data based on gamma ray densitometry," Meas. Sci. Technol., vol. 10, pp. 619-630, 1999.

[15] H. M. Prasser and U. Hampel, "Gamma and X-ray tomography for transient two-phase flows and other instrumentation developed by ROSSENDORF," in Proc. Progrès Récents des Methodologies de Modélisation des Écoulements Diphasiques, Lyon, France, Nov. 24-26, 2004.

[16] W. Hassan, S. Legoupil, and J. F. Delerue, "Tomosim: A virtual x-rays simulation code," 2005, submitted for publication.

[17] N. Freud, P. Duvauchellea, J. M. Létanga, and D. Babota, "Fast and robust ray casting algorithms for virtual X-ray imaging," Nucl. Instrum. Methods Phys. Res. B, Beam Interact. Mater. At., vol. 248, no. 1, pp. 175-180, Jul. 2006

[18] F. Tola, "Logiciels d'aide à la conception et à l'interprétation de mesures basées sur la détection d'un rayonnement $\beta \gamma$ ou X," DAMRI/SAR/S/96-123/T41, 1996, Commisariat à l'energie atomique, Grenoble, France. 